\title{
Profile of medical and injury consultations of Team South Africa during the XXVIIIth Olympiad, Athens 2004
}

\author{
Wayne E Derman (MB ChB, BSc (Med)(Hons), PhD, FACSM, FFIMS) \\ UCT/MRC Research Unit for Exercise Science and Sports Medicine, Sport Science Institute of South Africa, Cape Town
}

\begin{abstract}
Objective. This descriptive study was undertaken to document the nature of medical and injury consultations of the athletes and officials of the South African Team at the 2004 Olympic Games, and to provide data for planning future events.

Setting. South African medical facility, 2004 Olympic Games, Athens, Greece.

Methods. Total number of consultations and diagnoses were ascertained from medical logs and patient files which were completed daily by the members of the medical team. A retrospective clinical audit of medical records was then undertaken and the data were then compared with similar data collected during the Sydney 2000 Olympic Games. Acute and chronic-soft tissue (muscle strain, ligament sprain, tendon injury, contusion or laceration) and bony injury were analysed in terms of nature of injury, grading of severity and anatomical region injured.
\end{abstract}

Main outcome measures. Number of consultations due to medical complaints or injuries among athletes and officials.

Results. A total of 180 medical consultations were logged during the time in Athens while 348 consultations were logged in Sydney. The daily consultation rate was 6 per day in Athens compared with 13 per day in Sydney. In Athens, $84 \%$ of consultations were with athletes and the remainder $(16 \%)$ with officials - this was similar to Sydney. The most common medical complaints in Athens were dermatological (16\%), ENT (13\%), and respiratory $(8 \%)$ in nature, which contrasts with the profile of consultations in Sydney (ENT 18\%; neurological system 16\% and respiratory $16 \%$ ). Acute injury and chronic injury accounted for $26 \%$ and $14 \%$ of consultations respectively. In Athens, the most common acute

\section{CORRESPONDENCE:}

Professor Wayne Derman

UCT/MRC Research Unit for Exercise Science and Sports Medicine

Sport Science Institute of South Africa

Newlands 7700

Cape Town, South Africa

Tel: 27-21-659-5644

Fax: 27-21-659-5633

E-mail: wayne.derman@uct.ac.za and chronic injuries were soft-tissue injuries. The most common acute injury regions were the foot and ankle (25\%), upper leg $(17 \%)$ and knee $(17 \%)$. A total $77 \%$ of acute injuries were grade I, $17 \%$ grade II and $6 \%$ grade III in severity. The most common chronic injury regions were foot and ankle (32\%), lumbar spine $(32 \%)$, and shoulder $(11 \%)$. These injury profiles were similar to those documented in Sydney 2000.

Conclusions. Injury and illness complaints of the South African team were fewer in Athens 2004 compared with those documented during Sydney 2000. This can be attributed to local environmental conditions and travel across time zones. These data should be useful for planning medical services for future multicoded events. The analysis of the nature of consultations suggests that it should be a prerequisite for physicians travelling with a multi-coded events team to have broad knowledge of both medical and injury management of athletes. Specifically, a sound knowledge of the management of soft-tissue injury is an important prerequisite for the personnel of the medical team.

\section{Introduction}

The 2004 summer Olympic Games in Athens represented the 4th Olympic Games to which the South Africa sent an Olympic team since readmission to Olympic competition in 1992. In accordance with the number of athletes in the team and in keeping with International Olympic Committee (IOC) allocations and previous experience and recommendations, the medical team was assembled. ${ }^{1,2}$ As spaces on the medical team are very limited, the correct balance of medical service provision remains a difficult endeavour.

In total, the medical team comprised 11 members including 4 sports physicians, 6 physiotherapists and a sport psychologist. In contrast to Sydney 2000, none of the large teams in the South African squad were competing in remote cities and therefore all medical services were centralised.

This report describes the nature and profiles of the consultations for both the athletes and officials. The objectives of this report are therefore to examine the delivery of medical services and compare the profile of medical consultations over the last two Olympic Games, as well as provide data for planning of medical support to future multi-coded sports events of similar nature.

\section{Methods}

The medical records and histories of athletes were obtained through clinical evaluation opportunities during the work-up in the Operation Excellence programme (the preparation programme of the National 
Olympic committee of South Africa) and at preparation camps in the build-up to the Olympic Games. At each opportunity a hard-copy medical record was completed and the data transferred to an electronic database. This medical database was taken to Athens.

Athletes were defined as the members of the team engaged in competition and officials were defined as team or athlete coaches, team managers, team technical staff, administration officials, medical staff and National Olympic Committee members.

We used data from patient files and medical encounter forms to determine the use of the medical services. Data were collected over 30 days, starting when the team arrived in Athens (1 Sept) until the end of the Olympic Games (30 Sept). The nature of injury, medical complaint, or treatment was recorded on a specifically designed medical encounter form at the time of examination or treatment by the examining physician. The datasheets were collected and retrospective clinical audit of the encounter forms and patient files was performed.

The term 'injury' was defined as any complaint that required medical diagnosis and management. Acute injury was defined as any new injury that required medical or physiotherapy intervention and was subsequently graded I, II or III. Grade I injuries required on-field intervention but the athlete was able to continue competition or training, grade II injuries required that the athlete was removed from participation or training for less than 48 hours, and with grade III injures, the athlete was unable to participate in training or competition for more than 48 hours. Chronic injuries were defined as either preexisting injury or acute injury that subsequently required ongoing medical evaluation and management.

\section{Results}

\section{Olympic preparation programme}

Medical and scientific preparation of the team began in 2002 with some members of the potential Olympic squad joining Operation Excellence. Athletes underwent medical and scientific evaluations both regionally (between 2002 and 2004) and at two camps: the first in Pretoria in January and the second in Pretoria in August 2004, before departure for Athens.

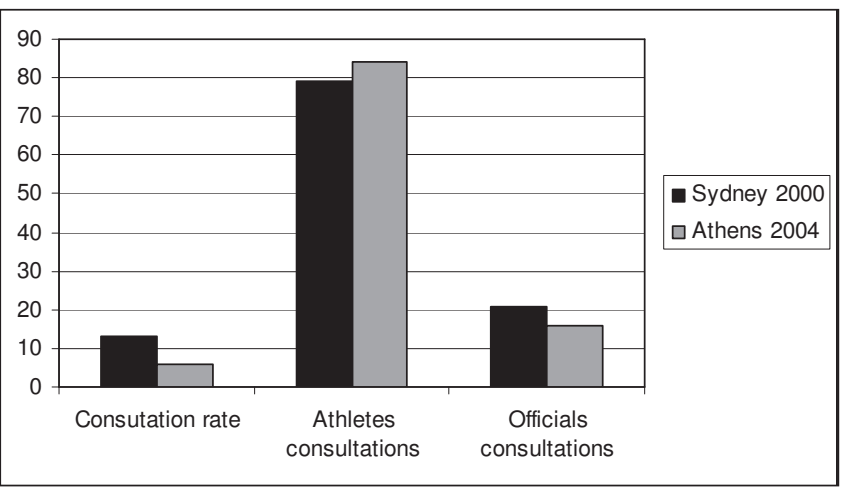

Fig. 1. Average daily consultation rate and relative percentage of total consultations for athletes and officials during the Sydney 2000 and Athens 2004 Olympic Games.
A total of 137 pre-participation medical assessments were conducted at these camps. The pre-participation programme included not only medical but also dietary, physiological, psychological and dental evaluations.

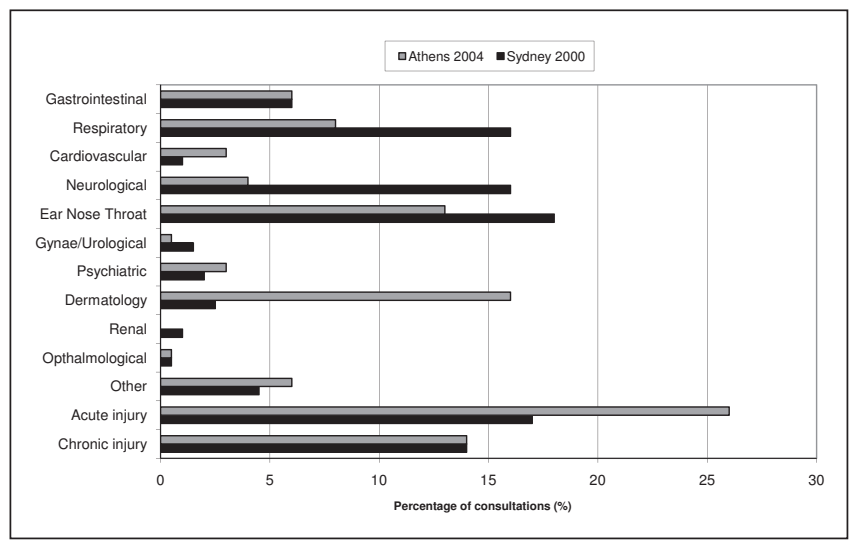

Fig. 2. Percentage of consultations due to injury or various medical complaints during the Sydney 2000 and Athens 2004 Olympic Games.

\section{Consultations and treatments during the Olympic Games}

In total 159 team members travelled to Athens. The team comprised 107 athletes from 18 sports codes and 52 officials; 180 formal medical consultations were conducted in the 30-day period. Of these consultations $84 \%$ (152 consultations) involved athletes and 16\% (28 consultations) involved officials. The rate of formal consultations averaged 6 per day. This figure does not include informal consultations by the sports physicians in the physiotherapy rooms, patients consulted at field-side, at pool-side, at the track or any other venue. The daily rate of consultations and relative distribution of consultations between athletes and officials for both Sydney 2000 and Athens 2004 is shown in Fig. 1. Relatively more consultations were conducted on a daily basis during Sydney 2000 compared with Athens 2004. The relative distribution of medical consultations between athletes and officials in Athens was similar to that documented in Sydney.

Of the consultations conducted with officials, $36 \%$ were due to injury mostly sustained through training with the athletes and $64 \%$ of consultations with officials were due to medical-related problems.

The nature of the main complaint requiring medical consultation with respect to the athletes is displayed in Fig. 2. In Athens 2004, 60\% of all consultations resulted from a medical (non-injury) complaint while $40 \%$ of all consultations were due to injury. This distribution of consultations was similar to that reported after Sydney $2000(69 \%$ non-injury related; $31 \%$ injury related). The main medical complaints noted during Athens 2004 were dermatological (16\%), ear, nose and throat (ENT) related (13\%); and respiratory related (8\%). These data contrast somewhat to those reported after Sydney 2000, where the main complaints were respiratory $(16 \%)$, neurological $(16 \%)$ and ENT (18\%) in nature.

Acute and chronic injury during Athens 2004 accounted for $26 \%$ and $14 \%$ of consultations respectively, which contrasts slightly with injury data from Sydney 2000 , where $14 \%$ and $17 \%$ of consultations were due to acute and chronic injury respectively. Data from 


\section{TABLE I. Acute injury by anatomical region during Athens 2004 Olympic Games}

\begin{tabular}{|c|c|c|c|c|c|c|c|c|}
\hline & Muscle strain & Ligament sprain & Tendon injury & Contusion & Laceration & Abrasion & Total & $\%$ \\
\hline Head \& neck & 0 & 0 & 0 & 0 & 2 & 0 & 2 & 6 \\
\hline Lumbar spine & 3 & 0 & 0 & 0 & 0 & 0 & 3 & 9 \\
\hline Hip \& pelvis & 1 & 0 & 0 & 0 & 0 & 0 & 1 & 3 \\
\hline Shoulder & 1 & 0 & 0 & 0 & 0 & 0 & 1 & 3 \\
\hline Upper limb & 0 & 0 & 0 & 1 & 1 & 1 & 3 & 9 \\
\hline Wrist \& hand & 0 & 0 & 0 & 2 & 0 & 0 & 2 & 6 \\
\hline Upper leg & 4 & 0 & 0 & 2 & 0 & 0 & 6 & 17 \\
\hline Lower leg & 1 & 0 & 0 & 0 & 1 & 0 & 2 & 6 \\
\hline Knee & 0 & 4 & 0 & 2 & 0 & 0 & 6 & 17 \\
\hline Foot \& ankle & 0 & 5 & 0 & 3 & 1 & 0 & 9 & 25 \\
\hline Total & 10 & 9 & 0 & 10 & 5 & 1 & 35 & \\
\hline$\%$ & 29 & 26 & 0 & 29 & 14 & 3 & & \\
\hline
\end{tabular}

\begin{tabular}{|c|c|c|c|c|c|c|}
\hline & Muscle injury & Ligament injury & Tendon injury & Bony injury & Total & $\%$ \\
\hline Head \& neck & 0 & 0 & 0 & 0 & 0 & 0 \\
\hline Lumbar spine & 3 & 3 & 0 & 0 & 6 & 32 \\
\hline Hip \& pelvis & 1 & 0 & 0 & 0 & 1 & 5 \\
\hline Shoulder & 0 & 0 & 2 & 0 & 2 & 11 \\
\hline Upper limb & 1 & 0 & 0 & 0 & 1 & 5 \\
\hline Wrist \& hand & 0 & 0 & 0 & 0 & 0 & 0 \\
\hline Upper leg & 1 & 0 & 0 & 0 & 1 & 5 \\
\hline Lower leg & 0 & 0 & 0 & 1 & 1 & 5 \\
\hline Knee & 0 & 0 & 1 & 0 & 1 & 5 \\
\hline Foot \& ankle & 0 & 4 & 0 & 2 & 6 & 32 \\
\hline Total & 6 & 7 & 3 & 3 & 19 & \\
\hline$\%$ & 32 & 37 & 16 & 16 & & \\
\hline
\end{tabular}

Athens 2004, detailing acute and chronic injury and the anatomical distribution of the complaint are listed in Tables I and II. The detailed data for these injuries from Sydney 2000 have been published in a previous edition of this journal. $^{2}$

Skeletal muscle strain injuries and ligamentous sprain injuries accounted for $29 \%$ and $26 \%$ of all acute injuries, while contusion injuries constituted $29 \%$ of all acute injuries (Table I). Lacerations and abrasion injuries accounted for the remainder of the acute injuries. The most common anatomical areas injured acutely were foot and ankle (25\%), knee (17\%) and upper leg (17\%). Lumbar spine injuries and upper limb injuries constituted $9 \%$ of acute injuries each. Altogether $77 \%$ of the acute injuries were classified as grade I injuries, $17 \%$ grade II and $6 \%$ were grade III injuries.

Chronic injuries evaluated during Athens 2004 are described in Table II. Muscle and ligamentous injuries accounted for $32 \%$ and $37 \%$ of the chronic injuries respectively, with tendon and bony injuries accounting for $15.5 \%$ of the injuries each. The most common anatomical sites injured were foot and ankle $(32 \%)$, lumbar spine $(32 \%)$ and shoulder $(11 \%)$ while the other anatomical regions listed accounted for the remaining $25 \%$ of injuries.

\section{Discussion}

With the exception of South African athletes living and training overseas, the vast majority of the athletes who travelled to Athens for the Olympic Games were examined during regional evaluations, and at the two pre-Olympic camps by the travelling team physicians. This allowed the team and the athletes to become familiar with each other before leaving to compete internationally. This also provided the South African athletes with opportunity for timeous medical intervention and identification, diagnosis and rehabilitation of underlying injury. Dietary and psychological evaluations as well as dental evaluations (and interventions if necessary), were also provided for the athletes as part of the preparation programme, particularly as dental problems have been shown to be common in this group of individuals. ${ }^{9}$ Medical records that were established during the preparation phase formed the base documentation records to be used in the Team South Africa medical facility in Athens.

The first important finding of this study was that there were on average 6 formal medical consultations per day during the Olympic Games in Athens. This figure contrasts with that of Sydney 2000, 


\section{er

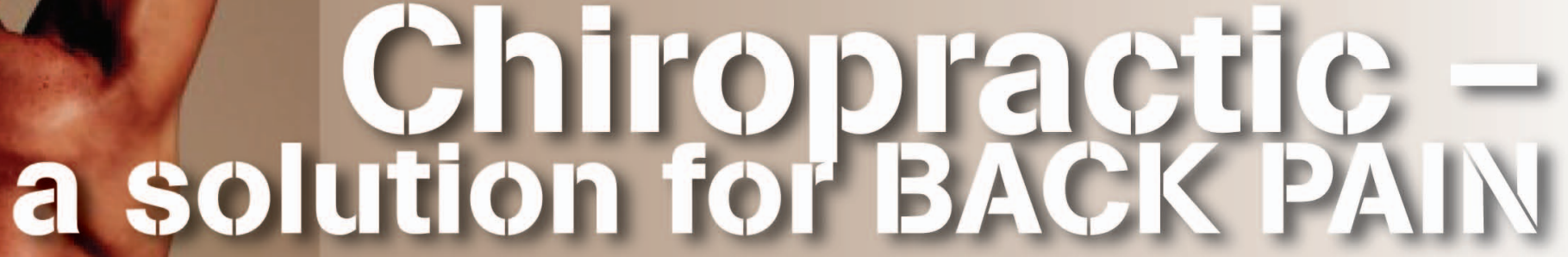 \\ Almost everyone EXPERIENCES back pain at some time but staying active with the help of chiropractic treatment may well be the best solution.}

Are you sitting comfortably?

Poor posture may lead to back pain. YourCASA Chiropractor may advise you to make postural changes if you:

- Spend hours a day sitting at a desk or a computer

- Slouch in front of the TV.

- Sleep in a bed that is too hard or soft

- Find yourself hunching your back and shoulders in stressful situations

"I simply leaned forward to brush my teeth"

Repeating daily activities such as bending, lifting and twisting may result in a "bad back". This is why your CASA chiropractor will want to understand how you tackle these everyday movements. He/She will also ask about any major traumas to your back such as:

- Car accidents

- Falls

- Sporting injuries

- Lifting heavy weights

- Unusual activities

Treating the cause, not the symptoms

As you go through life a slight loss of proper movements of the bones/joints can interfere with the healthy working of your spine and the nerves that pass through it. This can lead to pain. Chiropractic, unlike painkilling drugs treats the cause of pain not just the pain itself. Your CASA chiropractor will carry out a full examination (including
$X$-rays if necessary), and ask you questions about your posture, medical history and lifestyle, to try and discover the cause of your back pain and offer a diagnosis of your complaint.

Treatment may then begin, often with gentle specific adjustments (the chiropractic word for manipulation) done by hand, to free stiffjoints and remove spinal nerve irritation. This effective drug free treatment is generally painless, although you may feel some short-term discomfort if your back is very painful.

Your chiropractor may recommend ice or heat treatment and massage. Scientific research shows that you should remain active - prolonged bed rest weakens the bone and muscles and reduces your chances of a full recovery - so you may also be advised to do some gentle exercise to ease pain, and help your body to recuperate.

\section{For more information on Chiropractic or where to find a CASA Chiropractor:}

Visit the Chiropractic Association of South Africa website

www.chiropractic.co.za

or call us on 0583034571

e-mail: casa1@telkomsa.net \& drreg@mweb.co.za

With acknowledgement to the British Chiropractic Association
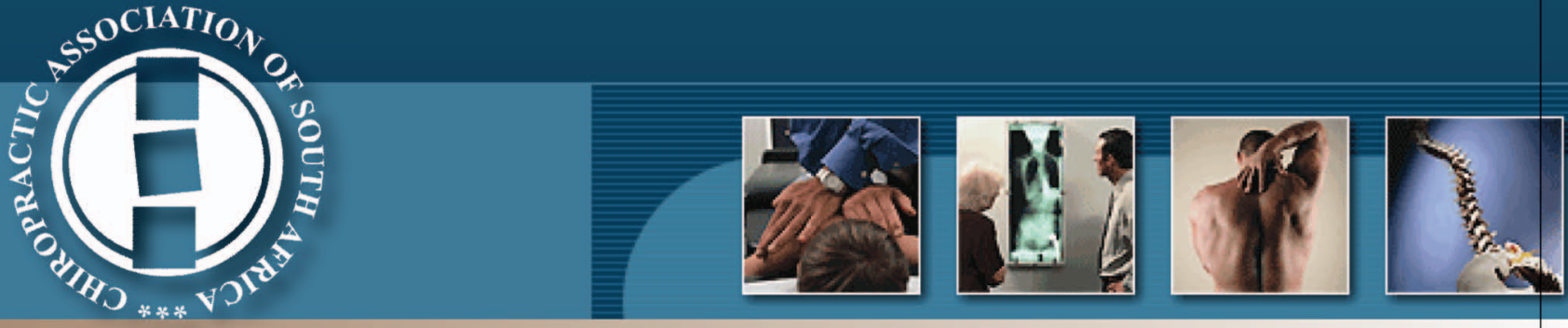
where the consultation rate was 13 per day. Indeed, during Athens 2004 only 180 formal medical consultations were conducted compared with 348 consultations over a similar period during Sydney 2000. Although it is possible that there might have been more 'informal' consultations that occurred field-side, pool-side and track-side, it is not possible to keep accurate records of all of these encounters. It is also possible that improved preparation and recovery strategies adopted by the athletes or improved screening and injury management might have accounted for the difference in consultation rate.

Yet, it is also of interest to note the differences in the profile of consultations depicted in Fig. 2. During Sydney 2000, many more consultations were conducted due to respiratory and ENT complaints, probably as a result of increased aero-allergens and subsequent symptoms associated with allergy, during spring time in Australia. ${ }^{3,5}$ Furthermore, during Sydney 2000 there were many more consultations in the first week of travel due to vague neurological complaints (symptoms of headache, malaise, insomnia), probably due to jet lag as a result of travel across many time zones. ${ }^{7}$ Although ENT-related complaints were among the most common encountered in Athens, the number of consultations was lower compared with Sydney 2000, due to different environmental conditions and improved identification and preparation of susceptible athletes. ${ }^{3}$ Furthermore, as travel to Athens did not involve crossing of many time zones, the team did not experience jet lag, which possibly explains the lower number of neurological complaints. The relative increase in consultations due to dermatological complaints was due to infection or allergic response secondary to ant and other insect bites, in and around the Athens Olympic village and fungal skin infections, probably as a result of the excessive heat and humidity that occurred in Athens at that time.

The second important finding of this study is that, as was the case in Sydney 2000, the majority of consultations were not due to injury. Although $40 \%$ of all consultations were due to sports injury, there were more consultations due to injuries in Athens than in Sydney. Indeed, relatively more of the injuries sustained in Athens 2004 were acute rather than chronic in nature, due to the fact that both men and women's hockey teams competed in Athens, while only the women's team competed in Sydney 2000. The anatomical distribution of these injuries at the two events was similar, and fortunately the vast majority of these injuries were of minor severity. The profile of the injuries and medical complaints described above is similar and comparable with those reported by the medical teams of other countries. ${ }^{1,4,10}$

Assessment of the injury and medical statistics allows for good future planning when considering the composition of a medical team for a multi-coded sporting event. It is clear that environmental conditions including airborne allergens, heat and humidity, travel across time zones and even insects in the immediate environment affect the profile of medical consultations during travel to sporting competition. It will be of interest to note the profile of consultations during the Olympic Games in Beijing 2008, where many of the abovementioned factors as well as high concentrations of air pollution were experienced. ${ }^{6,8}$

Due to the profile of consultations depicted in Fig. 2, team physicians should ideally have adequate experience with respect to both injury diagnosis and management and general sports medicine. Indeed, good skills with respect to ENT, respiratory, neurological and dermatological aspects of sports medicine are important. With respect to sports injuries, knowledge and skills in the management of musculo-skeletal (mostly soft-tissue) injury, particularly of the foot and ankle, lumbar spine and knee regions are important.

In conclusion, this study describes the medical care provided to the athletes and officials of the South African team to the Olympic Games in Athens 2004. Injury and illness complaints were fewer compared with those documented during Sydney 2000, probably due to local environmental conditions and travel across time zones. The analysis of the nature of consultations suggests that it should be a prerequisite for team physicians to have broad knowledge of both medical and injury management of athletes in order to accompany sports teams to multi-coded events. Furthermore, a sound knowledge of the management of soft-tissue injury, particularly in the foot and ankle, lumbar spine and knee regions, is an important prerequisite for the personnel of the medical team.

\section{Acknowledgements}

The author would like to thank Dr Christa Janse van Rensburg, Dr Harald Adams and Dr Maki Ramagole for their support and effort in collection of the data used in this study.

\section{References}

1. Budgett $R$, Harries $M$, Aldridge J, Jaques R, Jennings DE. Lessons learnt at the 1996 Atlanta Olympic Games. Br J Sports Med 1997; 31: 76.

2. Derman WE. Medical care of the South African Olympic Team -- the Sydney 2000 experience. South African Journal of Sports Medicine 2003; 15: $22-5$.

3. Hawarden D, Baker S, Toerien A, et al. Aero-allergy in South African olympic athletes. S Afr Med J 2002; 92: 355-6.

4. Junge A, Langevoort G, Pipe A, et al. Injuries in team sport tournaments during the 2004 Olympic Games. Am J Sports Med 2006; 34: 565-76.

5. Katelaris $\mathrm{CH}$, Carrozzi FM, Burke TV, Byth K. Patterns of allergic reactivity and disease in Olympic athletes. Clin J Sport Med 2006; 16: 401-5.

6. Li J, Lu Y, Huang $\mathrm{K}$, et al. Chinese response to allergy and asthma in Olympic athletes. Allergy 2008 ;63: 962-8.

7. Milne CJ, Fuard MH. Beating jet lag. Br J Sports Med 2007; 41: 401.

8. Milne CJ, Shaw MT. Travelling to China for the Beijing 2008 Olympic Games. Br J Sports Med 2008; 42: 321-6.

9. Piccininni PM, Fasel R. Sports dentistry and the olympic games. $J$ Calif Dent Assoc 2005; 33: 471-83.

10. Robinson D, Milne C. Medicine at the 2000 Sydney Olympic Games: the New Zealand health team. Br J Sports Med 2002; 36: 229. 